
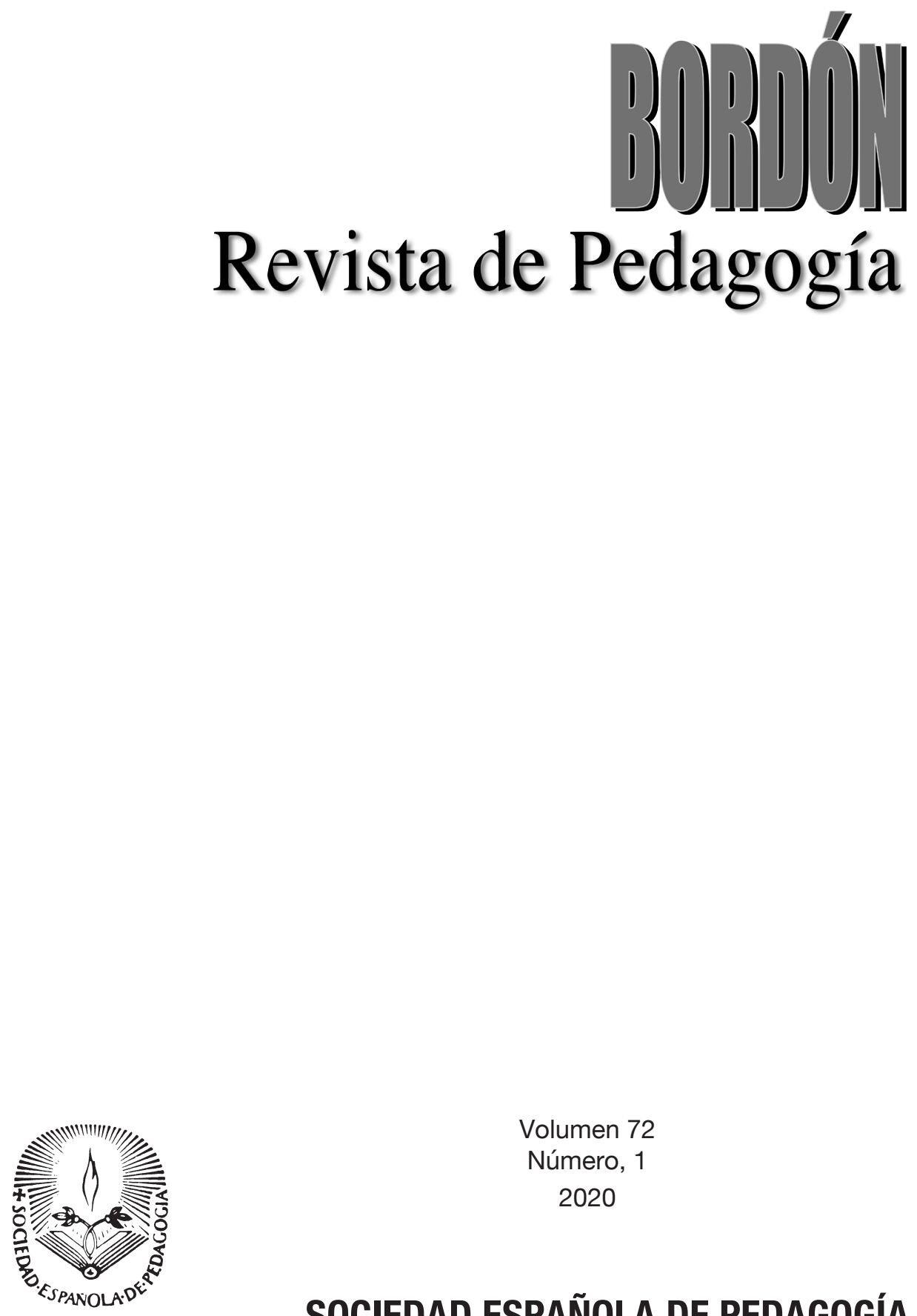

Volumen 72

Número, 1

2020 


\title{
BUEnAS PRÁCTICAS En LA ORGGnIZACIÓn, GESTIÓn Y DIRECCIÓn DE LOS CENTROS EDUCATIUOS
}

\section{Best practice in the organization, management, and direction of educational centers}

\author{
MANUEL CASTILLO GARCÍA(1), MANUEL JESÚS RAMOS CORPAS ${ }^{(2)}$ Y GONZALO DEL MORAL ARROYO(3) \\ (I) Universidad Hispalense de Sevilla (España) \\ (2) (3) Universidad Pablo de Olavide de Sevilla (España)
}

D01: 10.13042/Bordon.2020.01.63135

Fecha de recepción: 02/04/2019 - Fecha de aceptación: 27/10/2019

Autor de contacto / Corresponding author: Manuel Jesús Ramos Corpas. E-mail: mjramcor@upo.es

INTRODUCCIÓN. El objetivo de este trabajo es profundizar en el conocimiento de las buenas prácticas en el ejercicio de la dirección escolar considerando las siguientes variables: las relaciones entre profesorado y equipo directivo, los procesos de retroalimentación, los resultados que debe tener una buena práctica, la respuesta a situaciones novedosas, las variables a tener en cuenta para alcanzar los objetivos, las actitudes del buen director, las dinámicas de proyecto e innovación, el intercambio de prácticas docentes y, por último, los procesos de autoevaluación. Sobre la dirección escolar se han realizado numerosos estudios en las últimas décadas, aunque esta investigación aporta un nuevo enfoque como es el de las buenas prácticas en la dirección escolar. MÉTODO. La metodología es cualitativa basada en la Grounded Theory a partir de la información obtenida mediante grupo focal y posterior codificación y triangulación de datos, con la ayuda de Atlas ti 5.0. RESULTADOS. Los resultados se presentan apoyados con ilustraciones específicas atendiendo a cada una de las variables estudiadas. DISCUSIÓN. Se organiza igualmente atendiendo a las variables estudiadas y se pone de manifiesto, como aspectos más destacables, que las relaciones entre profesorado y equipo directivo tienen que ser respetuosas, horizontales, bidireccionales y participativas, desarrolladas mediante estrategias de cooperación y colaboración. Deben tener en cuenta procesos de retroalimentación mediante la potenciación de la cultura de la autoevaluación como motor de los procesos de mejora en el centro, y no solo deben conseguir buenos resultados, sino mejorar los anteriores; no obstante, deberá tenerse en cuenta el contexto de aplicación.

Palabras clave: Dirección escolar, Buena práctica, Retroalimentación, Innovación. 


\section{Introducción}

Los estudios sobre buenas prácticas educativas (en adelante, BB. PP.) han experimentado un gran impulso en las últimas décadas (Escudero, 2009; Álvarez, 2010; Pérez, 2011; Zabalza, 2012; Castillo, Amador, Ramos y Musitu, 2017). Entre ellos cabe destacar los realizados dentro del proyecto de investigación PIV066/2012 titulado Buenas prácticas en la organización y funcionamiento de institutos de enseñanza secundaria, aprobado por la Consejería de Educación de la Junta de Andalucía (Castillo y Ramos, 2012; Ramos y Castillo, 2013; Ramos, 2013; Carmona, Ramos y Castillo, 2014; Castillo, Ramírez y Ramos, 2015), cuyas conclusiones se tuvieron en cuenta en el equipo específico de conceptualización, recogida y difusión de BB. PP. docentes e inspectoras, constituido en el seno de la Inspección Educativa de Andalucía durante el curso 2015-2016, que llegó a establecer la siguiente definición, requisitos y condiciones: "Buena práctica docente es aquella experiencia innovadora que concreta un modelo de actuación que mejora tanto los procesos escolares como los resultados educativos que con ellos se obtienen, y debe ser innovadora, efectiva, sostenible y replicable" (UNESCO, 2009). También se establecieron seis condiciones: debe tratarse de una acción y no de intenciones; los objetivos deben estar definidos y ser pertinentes y realistas; el cumplimiento de la norma no puede considerarse buena práctica; no debe suponer un aumento de recursos ni materiales ni humanos, sino un mejor aprovechamiento de los mismos; debe responder a las necesidades del alumnado; y debe responder a las necesidades derivadas del contexto del centro.

Chickering y Gamson (1991) identifican siete principios que configuran una buena práctica educativa: promueve las relaciones entre profesores y alumnos; desarrolla dinámicas de cooperación entre los alumnos; aplica técnicas activas para el aprendizaje; permite procesos de retroalimentación; enfatiza el tiempo de dedicación a la tarea; comunica altas expectativas; y respeta la diversidad de formas de aprender.
Para Abdoulaye (2003: 3) las BB. PP. son "ejemplos exitosos de cambio en modos de hacer que mejoran un estado de cosas existente y que comportan una serie de criterios o estándares a los que se atienen". Marqués (2002), en su definición de BB. PP. docentes incluía requisitos, como facilitar el aprendizaje y ser eficientes. Epper y Bates (2004) propusieron algunas exigencias a la caracterización de las BB. PP., entre ellas: contribuir a mejorar el desempeño de un proceso; sistematización; estar documentadas y experimentadas; diseño innovador.

De Pablos y Jiménez (2007: 17) se refieren a las BB. PP. como un concepto que puede adquirir distintos usos y acepciones, entre ellos: una manera de modelizar y ejemplificar una actividad realizada con resultados satisfactorios; reflejo/ producto de la identidad de un contexto; el término de "buenas" le otorga carácter de exportabilidad.

Pérez (2011: 9) definía las BB. PP. como acciones "innovadoras, eficaces, sostenibles, contrastables, transferibles y fiables, integradas en un proceso previamente planificado, reflexivo y sistemático, que pretende dar respuesta a una necesidad sentida por los miembros de una institución o colectivo, orientado a conseguir los objetivos propuestos, en un contexto determinado".

Por su parte, Escudero (2009) establece distintas condiciones para definir una BP. Por un lado, debe ser exitosa y, por otro, debe haber posibilidad de replicación por parte de otros sujetos que pretendan alcanzar objetivos similares. No obstante, aunque los escenarios educativos no sean semejantes, las BB. PP. deberán estar validadas y poder ser transferidas a otras situaciones similares con las adaptaciones que en todo caso el profesional docente deberá valorar. Este tipo de consideraciones son avaladas por el Consejo Escolar de Estado al definir las BB. PP. como "expresión de un conocimiento profesional o experto, empíricamente válido, formulado de modo que sea transferible y, por 
tanto, de potencial utilidad para la correspondiente comunidad".

Pérez (2011) establece una serie de orientaciones para llevar a cabo las BB. PP., entre las que destacamos algunas consideraciones como: pretender dar siempre respuesta a una necesidad; responder a un modelo teórico; deben causar efecto sobre algo; tienen que ser innovadoras, eficaces, sostenibles, contrastables, transferibles y fiables; han de ser integradas en un proceso previamente planificado, reflexivo y sistemático. Así pues, nos situamos a medio camino entre el "deber ser" de los procesos marcados por el cumplimiento de normas o estándares vigentes y los modos de hacer más artísticos y situacionales (Zabalza, 2012).

Desde esta perspectiva, se necesitan elementos diferenciadores entre "lo bueno" y "lo malo". En educación, no estamos acostumbrados a la utilización de términos peyorativos; simplemente si una acción no da resultados sobre los educandos se procede a incorporar y adaptar los procesos. Según Cabré (2010: 1) "lo que se considera 'bueno' depende de quién lo juzgue, del objeto sobre el que se aplica, de dónde y con qué finalidad se aplica, o desde qué posición o ideología se actúa, por considerar solo algunas posibilidades".

Para el Ministerio de Educación, Cultura y Deportes (2014) "una buena práctica es una iniciativa, una política o un modelo de actuación exitoso que mejora, a la postre, los procesos escolares y los resultados educativos de los alumnos" (MECD, 2014).

La importancia de la dirección en la organización de los centros educativos se ha puesto de manifiesto por diversos autores e informes (Batanaz, 2006; Montero, 2012; Informe Mckinsey, 2010). La dirección escolar responde a la forma con la que la situación socio-política-educativa de un país haya evolucionado en el discurrir de los años. Mayorga (2007: 93) pone énfasis en la complejidad de dirigir un colegio: "la dirección escolar es una figura clave en la organización y funcionamiento de los centros educativos, que adquiere cada día mayor relieve debido a la creciente complejidad de las instituciones escolares".

La dirección escolar es un factor clave en la organización de los centros educativos, por lo que se han realizado numerosos estudios sobre diversos aspectos, entre los que destacamos estudios sobre el perfil de la dirección y sus funciones (Fullan, 2014; Serrano y Martín-Cuadrado, 2017), sobre la forma de elección y acceso (Montero, 2012; Rodríguez, Rodríguez, Artiles, Aguilar y Alemán, 2013), sobre cómo se podrían evaluar (Armas, 1996), sobre la necesidad de su profesionalización (Lorente, 2012; Barrios, Iranzo y Tierno, 2013), sobre su autoestima (Bernal, 1995), sobre su liderazgo y logros académicos (Bolivar, 2013; Blase y Kirby, 2013), sobre su formación y selección (Cantón, 2013; Silva, del Arco y Flores, 2017), sobre su evolución histórica (Murillo y Gómez, 2006) o sobre las dificultades para su ejercicio (Villa, 1998), pero no se han encontrado estudios que se centren en el enfoque de la detección de buenas prácticas en el ejercicio de la dirección de centros educativos. La presente investigación pretende satisfacer esta necesidad, por lo que desde la metodología cualitativa se ha querido profundizar en el conocimiento de las buenas prácticas en el ejercicio de la dirección escolar considerando las siguientes variables: las relaciones entre profesorado y equipo directivo, los procesos de retroalimentación, los resultados que debe tener una buena práctica, la respuesta a situaciones novedosas, las variables a tener en cuenta para alcanzar los objetivos, las actitudes del buen director, las dinámicas de proyecto e innovación, el intercambio de prácticas docentes y, por último, los procesos de autoevaluación.

En la actualidad, ningún organismo ha realizado estudios sobre los indicadores de BB. PP. tanto en la organización como en la dirección escolar. 
Un referente podrían ser los indicadores de evaluación de directores. En este sentido las Administraciones Públicas están desarrollando protocolos de evaluación.

\section{Método}

\section{Objetivos del estudio}

El presente estudio plantea dos objetivos generales. En primer lugar, identificar indicadores de BB. PP. educativas en la organización y dirección escolar atendiendo a las siguientes categorías de análisis: a) relaciones entre profesorado y equipo directivo; b) procesos de retroalimentación; c) resultados de una BP; d) respuesta a situaciones novedosas; e) variables a tener en cuenta para alcanzar objetivos; f) actitudes del director; g) dinámicas de proyecto e innovación; h) intercambio de prácticas docentes; e i) procesos de autoevaluación del centro educativo. En segundo lugar, analizar cada categoría de BB. PP. educativas en la organización y dirección escolar estableciendo marcos de referencia compartidos y aspectos para su mejora.

\section{Diseño y participantes}

Este estudio forma parte de un proyecto de investigación más amplio dirigido a describir BB. PP. en educación en 5 áreas diversas: dirección, organización y gestión de centros educativos; nuevas tecnologías; prácticas docentes; atención a la diversidad; y orientación, tutoría y relaciones con la familia (Ramos Corpas y Castillo García, 2013). Para llevar a cabo la presente investigación se optó por un diseño descriptivo-comprensivo de corte cualitativo siguiendo los pasos analíticos propuestos por la Grounded Theory (Glaser y Strauss, 1967) a partir de la información obtenida mediante grupos focales. La técnica de muestreo utilizada ha sido intencional teórica (Strauss y Corbin, 2007), siguiendo una serie de criterios maestros de selección (Valles, 2003; Singleton y Straits, 2004; Suárez, Del Moral y González, 2013), tal y como se muestra en la tabla 1.

Tabla 1. Variable, fuente y criterio de selección. Expertos

\begin{tabular}{|c|c|c|}
\hline Variable & Fuente & Criterio \\
\hline $\begin{array}{l}\text { Cargo ocupado } \\
\text { en el centro }\end{array}$ & $\begin{array}{l}\text { Establecido } \\
\text { por los } \\
\text { investigadores }\end{array}$ & $\begin{array}{l}\text { Miembro del } \\
\text { equipo directivo }\end{array}$ \\
\hline $\begin{array}{l}\text { Buena práctica } \\
\text { de dirección, } \\
\text { organización y } \\
\text { gestión }\end{array}$ & $\begin{array}{l}\text { Ficha de } \\
\text { recogida de } \\
\text { información } \\
\text { sobre BB. PP. } \\
\text { en los centros } \\
\text { educativos } \\
\text { (elaboración } \\
\text { propia) }\end{array}$ & $\begin{array}{l}\text { Haber realizado } \\
\text { una BB. PP. } \\
\text { de dirección, } \\
\text { organización y } \\
\text { gestión en su } \\
\text { centro educativo }\end{array}$ \\
\hline Etapa educativa & $\begin{array}{l}\text { Establecido } \\
\text { por los } \\
\text { investigadores }\end{array}$ & CEIP, IES \\
\hline
\end{tabular}

El proceso de muestreo se realizó en dos fases. En la fase inicial se recabó información sobre las BB. PP. llevadas a cabo en centros escolares y para ello se diseñó un instrumento denominado "ficha de recogida de información sobre BB. PP. en los centros educativos" que fue enviado a un total de 152 centros educativos de la provincia de Sevilla. Esta herramienta consta de dos partes: en la primera se recogen los datos identificativos del centro escolar (identificador que garantiza el anonimato del centro, tipo y zona geográfica), y en la segunda se solicita al centro que realice 5 acciones: clasificación de la BP en una serie de categorías y subcategorías; descripción y justificación de la BP; identificación de los objetivos que pretende; redacción de los resultados obtenidos; y redacción de las conclusiones sobre la BP. El proceso de construcción y selección de categorías de BB. PP. se recoge en un trabajo anterior (Castillo y Ramos, 2012) en las que en principio se había estructurado la funcionalidad de los centros (ver ilustración 1). 
ILUSTRACión 1. BB. PP. analizadas en el proyecto de investigación amplio

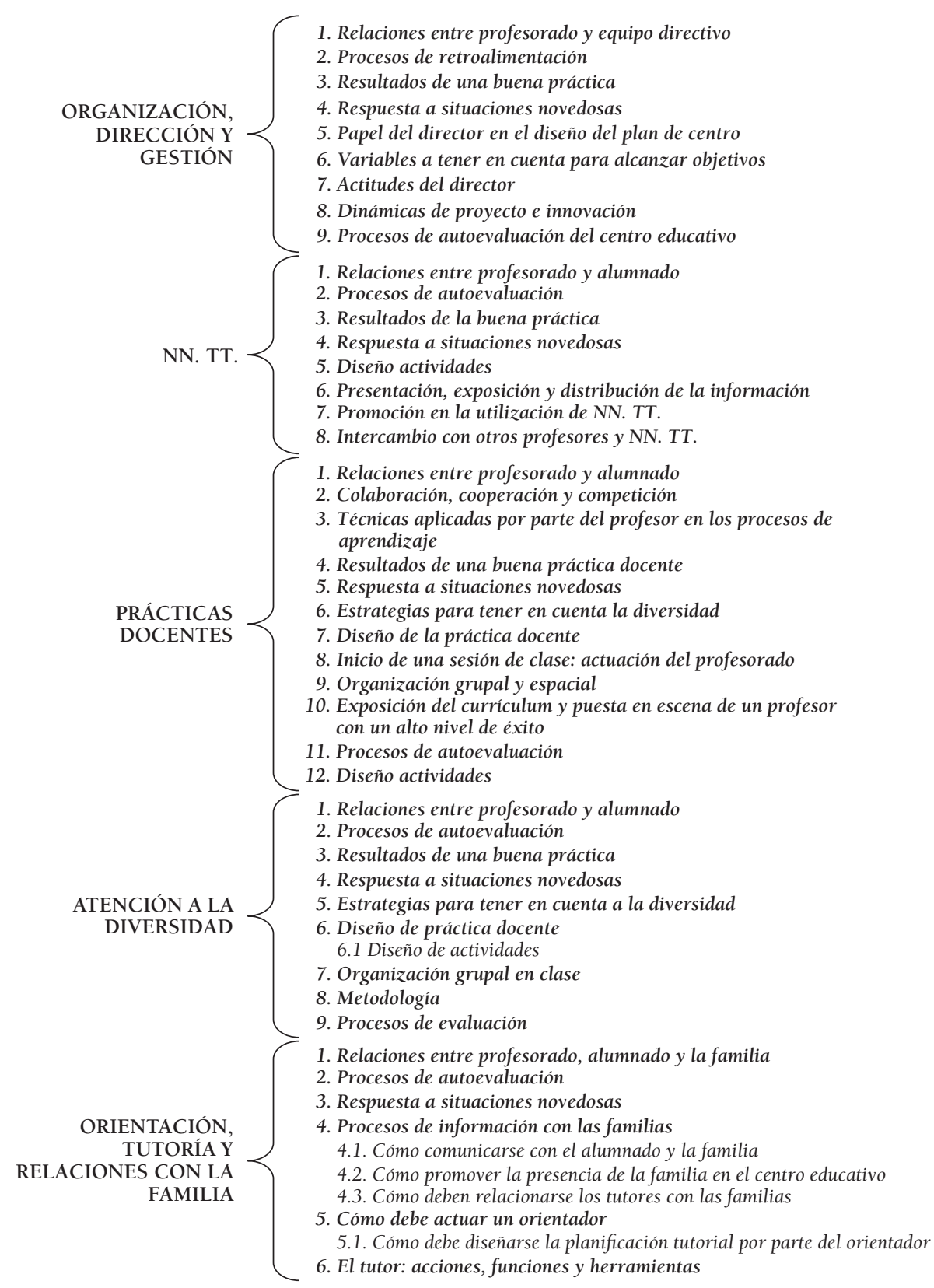

Fuente: elaboración propia.

En la segunda fase del muestreo, se seleccionaron aquellas BB. PP. que pertenecían a la categoría "buena práctica de dirección, organización y gestión de centros educativos", obteniéndose un total de 8 centros ( 4 IES y 4 CEIP) y 8 representantes de los mismos ( 7 directores y 1 jefe de estudios), siendo imprescindible que se tratara de algún miembro del equipo directivo. El 
100\% de los mismos aceptaron la participación en la siguiente fase del estudio: la realización de un grupo focal. Merece ser subrayado que una de las virtudes de esta investigación es que los resultados obtenidos surgen de la reflexión de personas expertas que, en el momento de la realización de este estudio, desempeñaban su labor como directivos o jefes de estudio y contaban con una gran experiencia acumulada.

\section{Estrategia de obtención de información y análisis de datos}

Para la obtención de información se utilizó la técnica del grupo focal, dirigida por dos moderadores expertos en BB. PP. siguiendo el formato de entrevista semiestructurada con un mismo guion en todos los grupos. Se realizó un único encuentro de 1 h 47 min de duración en uno de los centros de la muestra. Se realizaron las preguntas representadas en la tabla 2, de acuerdo con el objetivo de la investigación.

No se utilizaron observadores durante las entrevistas. No se atendió a la información no verbal, registrándose únicamente información en audio mediante grabadora Olympus DS-75, previo consentimiento explícito de los integrantes de los grupos. Se informó a todos los participantes del tratamiento de los datos, del consentimiento para reflejar el centro educativo y el cargo a la hora de publicar los resultados y la posibilidad de abandonar el estudio en cualquier momento. Los discursos recogidos fueron trascritos posteriormente a texto por el investigador principal con la colaboración de un segundo investigador. Los documentos se archivaron electrónicamente en formato enriquecido (.rtf) para facilitar su análisis posterior con Atlas ti 5.0.

\section{Análisis de la información}

Por último, para el análisis de la información recogida desde el enfoque de la Grounded Theory se realizaron tres tareas fundamentales de codificación: codificación abierta, codificación axial y codificación selectiva. La primera de ellas se encuadra dentro de la dimensión descriptiva de los datos, mientras que las dos últimas forman parte de la dimensión explicativa del proceso.

\section{Tabla 2. Preguntas llevadas a cabo en el grupo focal}

1. ¿Cómo deben ser las relaciones entre profesores y equipo directivo?

2. ¿Entre las distintas dinámicas de competición, colaboración o cooperación, existe alguna que se pueda considerar como idónea?

Preguntas generales $\quad 3$. ¿El equipo directivo permite procesos de retroalimentación respecto al funcionamiento del centro? ¿Cómo? ¿Qué hace?

4. ¿Influyen a su juicio los resultados para que una práctica se considere "buena"? ¿De qué tipo?

5. ¿Cómo debe responder una BP a situaciones novedosas?

6. ¿Qué papel desempeña un director en la elaboración del diseño del currículo de un centro? (programa educativo)

7. ¿Qué tipo de variables respecto a la organización y gestión de un centro debería tener en cuenta un equipo directivo con el propósito de alcanzar los objetivos del centro?

Preguntas específicas 8. ¿Cómo tiene que tener en cuenta la participación del profesorado?

9. ¿Qué actitudes debería tener un director?

10. Respecto a la dinámica de proyectos e innovación, intercambios de prácticas... ¿Cómo debe actuar un director?

11. Respecto a la propia evaluación del centro, para la mejora, ¿qué debe hacer un equipo directivo? 
a) Codificación abierta: se identificaron y definieron fragmentos significativos del texto, los cuales fueron codificados de manera intuitiva, abierta y sin presupuestos teóricos prefijados.

b) Codificación axial: se analizaron relaciones entre conceptos que definían los vínculos entre categorías y subcategorías para establecer categorías principales y secundarias.

c) Codificación selectiva: las categorías y subcategorías se redujeron e integraron en redes conceptuales descriptivas o explicativas con apoyo de la literatura previa.

En el presente estudio se ha realizado un proceso de codificación secuencial deductivo e inductivo. En primer lugar, se ha partido de un sistema de códigos teóricos previos que serían temas y subtemas que a criterio de los investigadores sería necesario considerar en el análisis de las BB. PP. educativas en los distintos grupos de discusión. Estos códigos teóricos estaban prefijados de antemano, aunque por supuesto sujetos a posibles cambios y modificaciones atendiendo al discurso de los participantes en los grupos de discusión. El proceso de construcción y selección de los primeros códigos teóricos de BB. PP. se basó en las dimensiones de categorización de Chickering y Gamson (1991): a) promueve las relaciones entre participantes; b) desarrolla dinámicas de cooperación; c) aplica técnicas activas; d) permite procesos de retroalimentación; e) enfatiza el tiempo de dedicación a la tarea; f) comunica altas expectativas; g) respeta la diversidad; y Alexander (1997): a) dimensión política; b) dimensión evaluativa; c) dimensión empírica; d) dimensión pragmática; y e) dimensión conceptual.

La estrategia analítica seguida consistió en transformar en códigos teóricos las propuestas de Chickering y Gamson (1991) y Alexander (1997), llevando a cabo un primer trabajo analítico deductivo, para continuar con una aproximación inductiva abierta mediante la cual poder codificar fragmentos significativos de información. Del mismo modo se utilizaron como códigos teóricos las distintas dimensiones de análisis de BB. PP. de organización, dirección y gestión propuestas por Castillo (2015): a) relaciones entre profesorado y equipo directivo; b) procesos de retroalimentación; c) resultados de una BP; d) respuesta a situaciones novedosas; e) variables a tener en cuenta para alcanzar objetivos; f) actitudes del director; g) dinámicas de proyecto e innovación; h) intercambio de prácticas docentes; e i) procesos de autoevaluación del centro educativo.

Una vez identificados estos códigos teóricos en los fragmentos significativos de la información transcrita se procedió a codificar el resto de citas de una manera abierta, intuitiva y sin presupuestos teóricos prefijados, es decir, no se pretendió validar ningún supuesto modelo teórico previo sobre buenas prácticas educativas, sino que el objetivo analítico inicial fue el de acercarse de una manera inductiva a las descripciones y explicaciones de los participantes sobre los temas y subtemas propuestos. Operativamente hay dos indicadores que se han tenido en cuenta para la selección de códigos analíticos que pasarían a ser tratados como categorías descriptivas/explicativas (es decir, este sería el paso fundamental del nivel textual al nivel conceptual): la fundamentación (grounded) o número de veces que aparece un código en un mismo texto y en distintos textos; y la densidad, que sería el número de relaciones que mantiene ese elemento con otros (ya sean otros códigos o anotaciones teóricas). Puede observarse un ejemplo del proceso en la ilustración 2.

Con el fin de garantizar la validez y el rigor de los resultados obtenidos se han utilizado tres estrategias analíticas. En primer lugar, la triangulación intradatos, o método comparativo constante (Strauss y Corbin, 1998), consistente en la comparación sistemática de las categorías y sus propiedades derivadas del análisis de los datos en distintos fragmentos de un mismo material, entre diferentes materiales y entre distintos sujetos, para buscar regularidades o pautas que permitan definir una categoría, propiedad o relación derivada del análisis de datos como significativa. 
Ilustración 2. Mapa de familia de códigos para la categoría "actitudes del director" en BB. PP. de organización, dirección y gestión

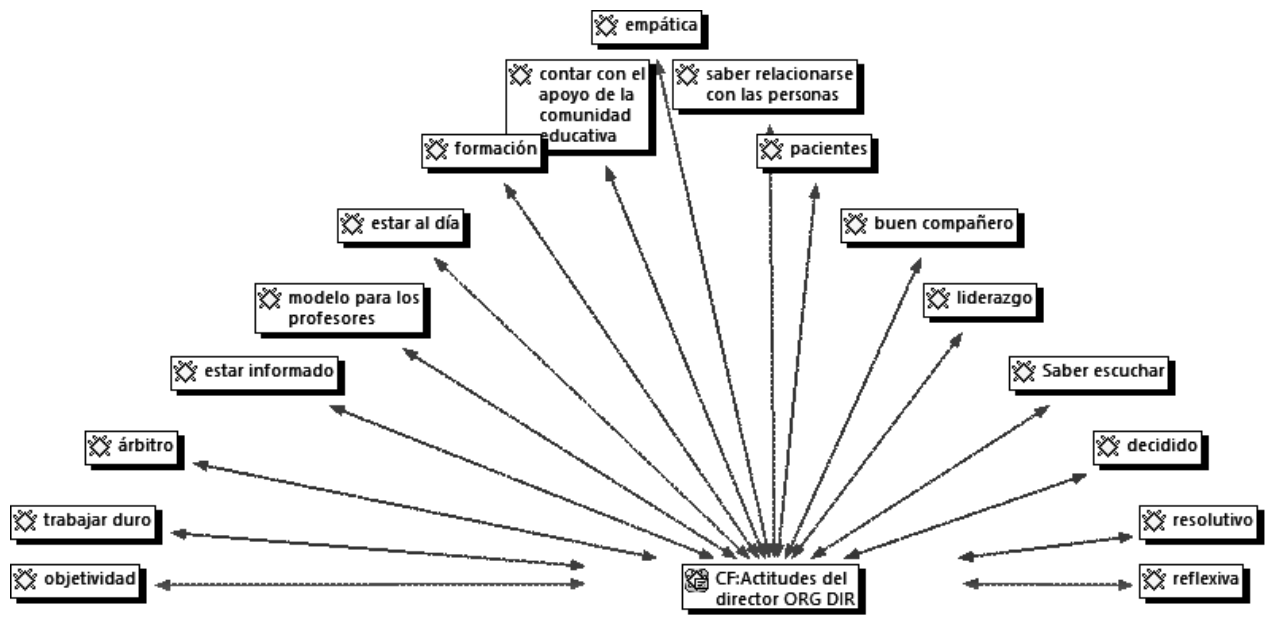

Fuente: elaboración propia.

En segundo lugar, la revisión por pares (Creswell y Miller, 2000; Thomas, 2011) consistente en contrastar los resultados de la codificación de cada investigador, descartando aquellos en los que no se lograra un acuerdo. Para ello se utilizó la herramienta informática Intercoder Reliability Calculator Recal2 (http://dfreelon.org/utils/recalfront/recal2/), que es una utilidad online que permite calcular los coeficientes de fiabilidad interjueces para datos nominales $u$ ordinales codificados por dos analistas mediante el estadístico alfa de Krippendorff. Un valor igual a 1 implicaría que los codificadores interpretan el código y realizan el análisis de manera idéntica. Valores entre 0.8 y 1 indicarían un buen grado de acuerdo interjueces. En el presente estudio se obtuvo un alfa de Krippendorff de 0.9. Finalmente, la literatura técnica ha servido en la fase de escritura para extender, validar y refinar el conocimiento del objeto de estudio (triangulación teórica; Olsen, 2004).

\section{Procedimiento}

Las fases llevadas a cabo en este estudio se explican a continuación: a) Envío de instrumentos. En primer lugar, se procedió al envío de la "ficha de recogida de información sobre BB. PP. en los centros educativos" mediante correo electrónico. Se asignó un tiempo de seis meses para la elaboración y reenvío del instrumento por parte de los docentes o equipos directivos que en principio estuvieran desarrollando BB. PP. en el centro escolar, ajustadas a cada una de las categorizaciones que se establecían en el instrumento al objeto de ser analizado posteriormente.

b) Recogida de información de los centros educativos y clasificación de las BB. PP. en función de las categorías y subcategorías descritas en que se estructuró la investigación (ver ilustración 1). No se establecieron filtros ni selección de las mismas en esta fase, no obstante, sí hubo un primer análisis sobre la calidad y cantidad de información suministrada por los centros, por lo que hubo una segunda fase en la que se le requirió a los centros el aporte de documentación complementaria, la cual se incorporó a la información general una vez recepcionada. De los 152 centros educativos a los que se mandó el 
instrumento, se obtuvieron 52 fichas sobre BB. PP. en las distintas categorías correspondientes a 23 centros educativos.

c) Selección de BB. PP. por categorías. Una vez identificadas las experiencias educativas que resaltaban alguna buena práctica se procedió a su selección, indagando sus aportes y características.

d) Asignación por categorías a grupos focales. De acuerdo con las categorías establecidas en el marco de los distintos procesos y ámbitos que se dan en un centro escolar, los investigadores asignaron las prácticas a las categorías establecidas.

e) Propuesta de participación en grupo focal para la obtención de información relevante. Para cada una de las distintas categorías anteriores, se seleccionaron un conjunto de prácticas y experiencias adaptadas a las categorías de la investigación para la organización de grupos focales mediante la invitación de los representantes o agentes que estuvieran desarrollando las mismas. f) Recogida de datos de los grupos focales en centros de la provincia.

g) Tras una planificación ajustada tanto a la necesidad de la investigación como a la disponibilidad del profesorado y los profesionales que habían aportado las fichas de BB. PP., se tuvo que realizar un plan de trabajo adaptado y que respondiera a las distintas restricciones que imponían una constante flexibilidad (restricciones de espacio, lugar de celebración, horarios del profesorado destinado en la provincia de Sevilla y las necesidades personales de cada participante). Se planificaron varios días y distintos centros de la provincia de Sevilla para llevar a cabo el desarrollo de los grupos focales.

\section{Resultados}

A continuación se presentan los principales resultados atendiendo a cada una de las variables contempladas en el objetivo de esta investigación:

ILUSTRACIÓn 3. Relaciones profesorado-equipo directivo

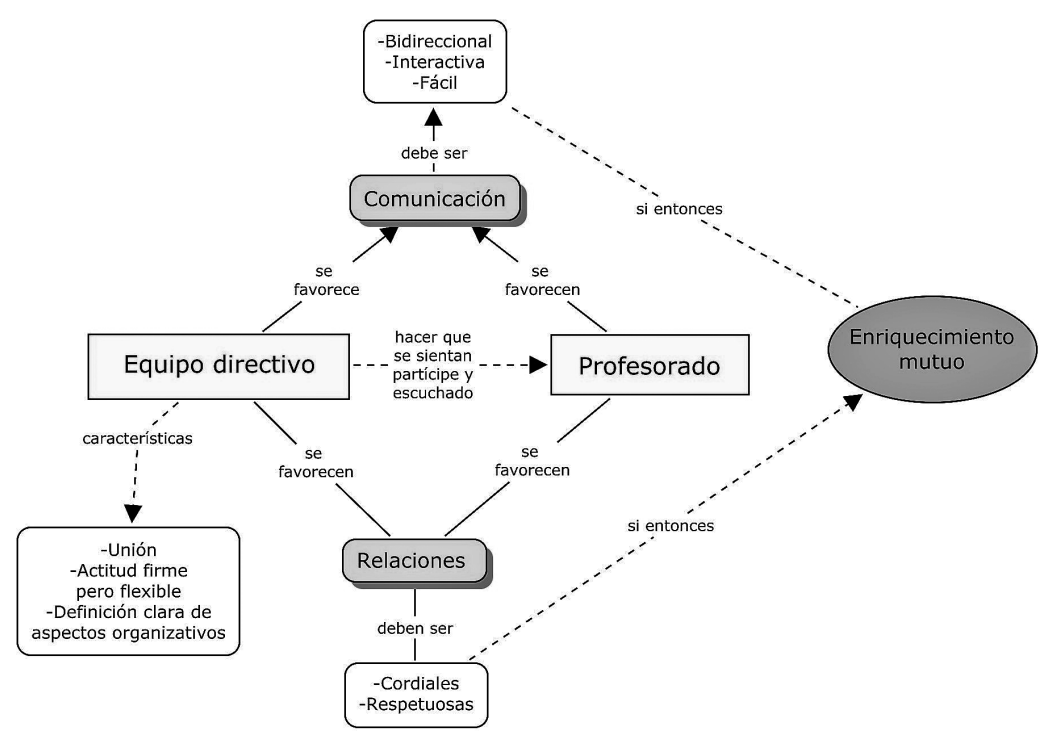

Fuente: Castillo et al. (2017). 
Relaciones entre profesorado y equipo directivo

Los participantes proponen que el equipo directivo debe mostrarse unido y con una actitud firme pero a la vez muy flexible, en la que el profesorado pueda encontrar muy bien definido el plan del centro, la normativa, los proyectos, los roles y funciones, etc. Es muy importante favorecer la participación del profesorado y recoger sus propuestas. Hubo consenso respecto a considerar la participación del profesorado como palanca para la innovación, la mejora del clima y, además, se posiciona como eje principal en la resolución de los conflictos del centro educativo.

\section{Procesos de retroalimentación}

Los participantes describen los procesos formales y organizativos de retroalimentación en los centros destacando una figura por encima de las demás: el equipo técnico de coordinación pedagógica (ETCP). Este equipo recoge las sugerencias, ideas de mejora, problemas, etc., del profesorado de los distintos ciclos y hace llegar esta información al equipo directivo. Los participantes incluyen también a los equipos de ciclo, los equipos docentes, las reuniones de tutoría, el claustro y el consejo escolar como el resto de figuras formales en la organización del centro que permiten que todo el mundo de sienta partícipe y escuchado. Se incluye en este proceso de retroalimentación a los padres y madres, a través de sus representantes en el consejo escolar.

Se hace referencia igualmente al uso de las NN. TT. como herramienta para recoger esta retroalimentación entre el profesorado (uso de $e$ mails y documentos colaborativos).

\section{Resultados de una BP}

Las acciones emprendidas deben mejorar los resultados en un área concreta para el que la BP se

\section{Ilustración 4. Procesos de retroalimentación en BB. PP. de organización, dirección y gestión}

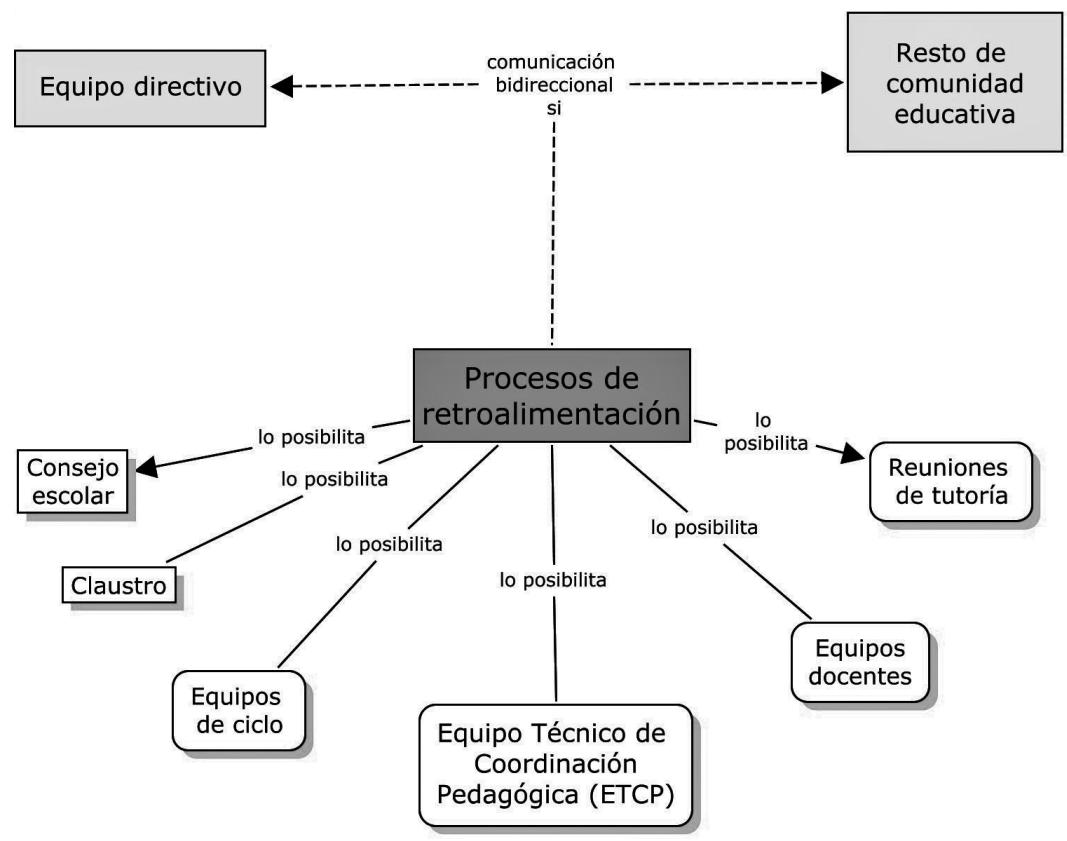

Fuente: Castillo et al. (2017). 
ILUSTRACIÓN 5. Resultados de buena práctica de organización, dirección y gestión

Fuente: Castillo et al.(2017).

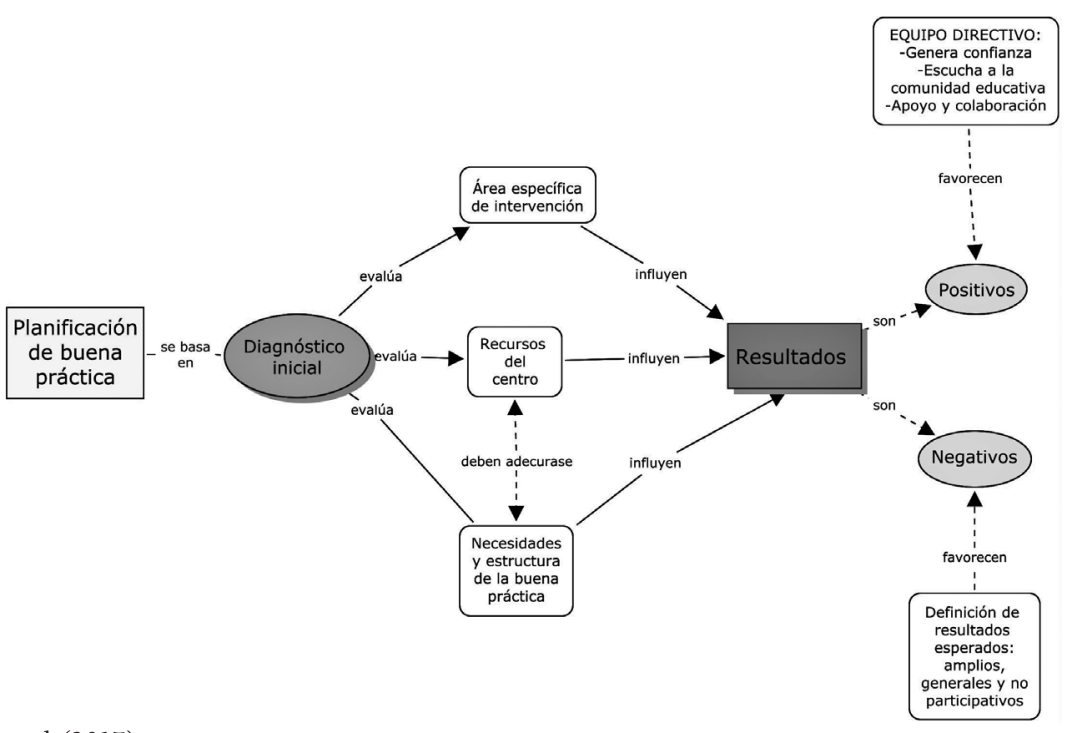

propuso (curricular, convivencial, relaciones entre el profesorado, etc.). Es decir, la BP no solo debe conseguir buenos resultados, sino mejorar los resultados anteriores. El concepto de "mejora de resultados" se configura, de igual forma, como dimensión no limitada por la amplitud de variables consideradas en los procesos escolares. Hablamos de procesos, competencias, relaciones, aptitudes, etc., y no solo de mejora de resultados en las calificaciones del alumnado. Destacan igualmente que la esencia de una BP de organización, dirección y gestión de centros educativos es como sigue: canalizar, organizar y dirigir teniendo en cuenta cuatro grandes áreas: profesorado, alumnado, familias y convivencia.

\section{Respuesta a situaciones novedosas}

Hay una categoría que emerge como una especie de límite o punto de referencia a partir del cual poder actuar: el marco normativo-legislativo. La respuesta a situaciones novedosas exigen, como otro

ILUSTRACión 6. Respuesta a situaciones novedosas BB. PP. de organización, dirección y gestión

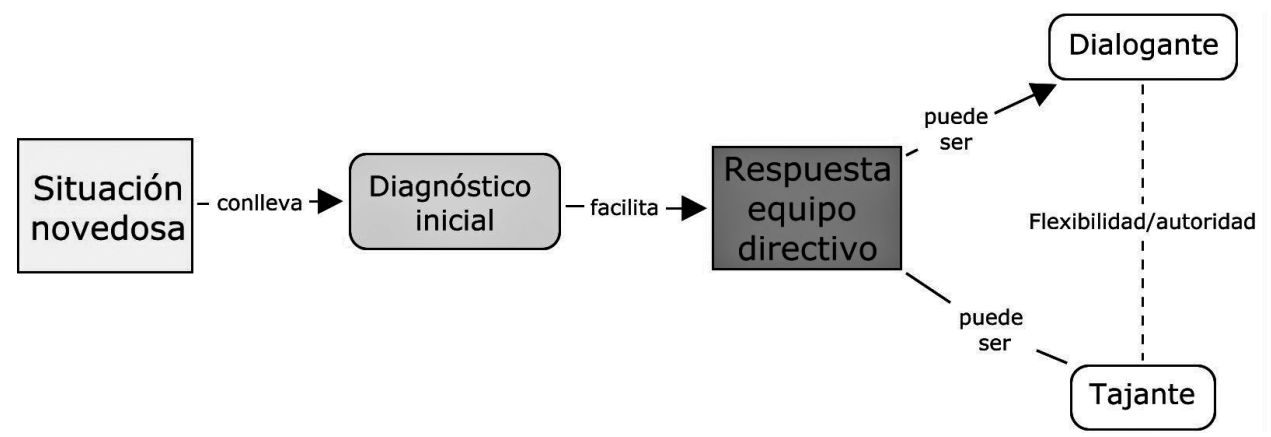

Fuente: Castillo et al. (2017). 
tipo de situaciones, un profundo diagnóstico inicial a partir del cual seguir dos caminos: uno que se puede denominar "dialogante", en el que la situación (que en este grupo focal se identifica con "problema") se puede resolver aplicando el sentido común por parte del equipo directivo y encontrando una solución dialogada; o por otro lado, existe la vía "tajante" en 1 a que la solución se encuentra a través de la aplicación de la ley y del ejercicio del cargo por parte del director y su equipo. En ambos casos, se incide en la importancia de pensar en el bien del centro educativo y valorar la dimensión flexibilidadautoridad.

Surgen algunas propuestas generales para afrontar situaciones novedosas: generar un clima de confianza y la actualización y formación del equipo directivo para estar al día.

\section{Variables a tener en cuenta para alcanzar objetivos}

Manifiestan la importancia del conocimiento profundo inicial del contexto al que pertenece el centro y de los recursos con los que se cuenta, en concreto: el claustro de profesores y variables como su estabilidad/continuidad, el personal ordenanza secundario, el alumnado del

\section{ILUSTRACIÓN 7. Variables a tener en cuenta para alcanzar objetivos en BB. PP. de organización, dirección y gestión}

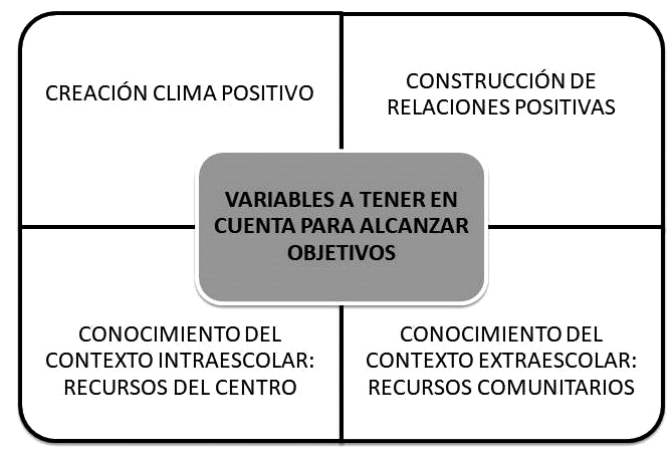

Fuente: Castillo et al. (2017). centro, sus familias, y el contexto comunitario (tipo de barrio, instituciones que trabajan en la comunidad, etc.). Este conocimiento debe servir para construir relaciones positivas que creen un clima de centro en que todos los miembros de la comunidad educativa se sien$\tan$ bien.

\section{Actitudes del director}

Hubo gran consenso respecto a las principales características que debe tener un director a nivel actitudinal: debe ser buen compañero, con capacidades mediadoras, empático, reflexivo, objetivo, paciente, resolutivo, que sea un modelo de conducta para el resto de la comunidad educativa, que muestre una actitud positiva hacia el trabajo, que esté informado y que esté al día sobre lo que sucede en su centro.

\section{Dinámicas de proyecto e innovación}

La labor principal del director en este campo es la de promocionar y fomentar los proyectos e ideas innovadores, divulgándolos con claridad, con el fin de que se sume la mayor parte del profesorado. Para ello es muy importante que sepa vender el proyecto, no imponerlo, y que crea realmente en él, que el resto de la comunidad educativa perciba que el director participa y se implica en ese proyecto, con una actitud de apertura a las propuestas realizadas por el equipo docente o cualquier otro miembro de la comunidad educativa, apoyándolas cuando sea necesario.

\section{Intercambio de prácticas docentes}

Los participantes proponen dos tipos de intercambio: a) intercambio de prácticas docentes intracentro: es necesario que el director conozca de primera mano la labor que está llevando a cabo el profesorado de su centro, para así poder difundir, promover y apoyar las 


\section{ILUSTRACIÓN 8. Intercambio de práctica docente: tipos y espacios}

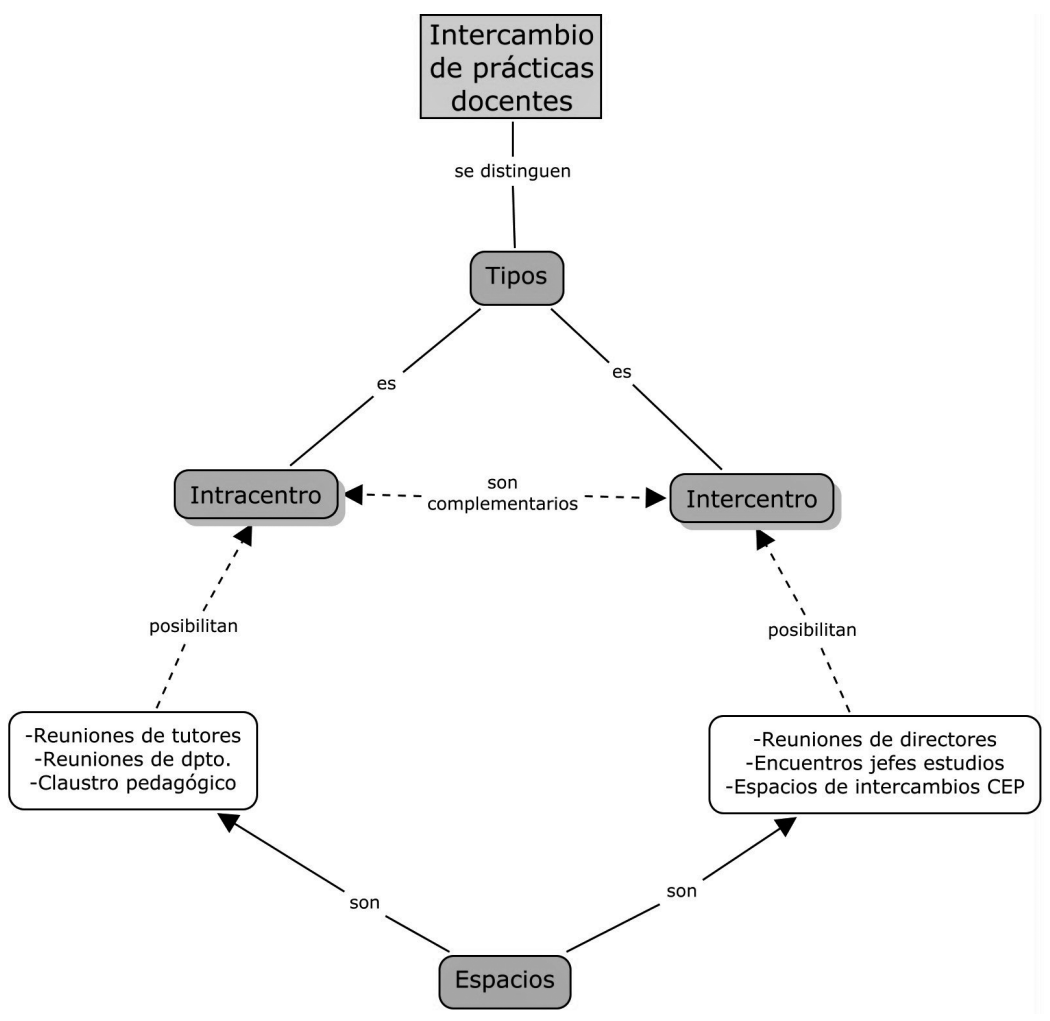

Fuente: Castillo et al. (2017).

BB. PP., dándolas a conocer al resto de la comunidad educativa. Los espacios que se proponen para este intercambio intracentro son: reuniones de tutores, reuniones de departamento y el claustro pedagógico, una herramienta muy útil que destacan varios participantes; y b) intercambio de prácticas docentes intercentro: de nuevo se destaca la importancia de este tipo de actuaciones (metabuenapráctica: BP para hablar de BB. PP.).

\section{Procesos de autoevaluación del centro educativo}

La autoevaluación como reflexión de lo que se está haciendo y de los resultados que se alcanzan respecto a los objetivos planificados constituye uno de los grandes problemas para los integrantes de los grupos. Coinciden en que el director será el órgano que debe impulsar y fomentar este tipo de prácticas, destacando su utilidad, proponiendo los participantes la descentralización de este proceso y dejándolo en manos expertas, por ejemplo, del Departamento de Formación, Innovación y Evaluación o de algún miembro del equipo docente experto. Destaca la separación existente entre la autoevaluación de aspectos organizativos y de funcionamiento versus los aspectos pedagógicos. Según los participantes, se hace poca autocrítica de los propios procesos pedagógicos por parte del profesorado, situando la génesis de los problemas relacionados con los resultados en factores externos (locus de control externo) como, por ejemplo, la falta de colaboración de las familias, la tipología de alumnado, etc. 


\section{Discusión}

Tras haber aplicado la metodología descrita, la discusión se organiza en las nueve variables del estudio:

\section{Relaciones entre profesorado y equipo directivo}

Un primer aspecto que nos parece interesante destacar es la idea de que las relaciones que se establecen en el marco de la organización escolar deben ser respetuosas; esto trae consigo que al mismo tiempo sean bidireccionales, respondiendo a la horizontalidad necesaria para la resolución de problemas y conflictos varios que cada día se dan en los escenarios escolares en los que no hay respuestas uniformes para la mayoría de los conflictos, ya que son diferentes. Estos resultados son coincidentes con otros donde se subraya de manera reiterativa la importancia de la bidireccionalidad, la comunicación y las relaciones; pero queremos enfatizar el valor del respeto como principal desencadenante o el motor de los procesos y relaciones entre los profesores y el equipo directivo (Murillo, 2008; Álvarez, 2010). El profesorado debe participar en la toma de decisiones del centro, en las normas y proyectos que emanan del centro. A su vez este aspecto revierte en el clima, en la confianza y el acercamiento al equipo directivo, conformando un sistema que redunda en la mejora de la escuela (Colom, 1970; Bertalanffy, 1976; Aracil, 1986) y por consiguiente en el aprendizaje de los alumnos. Nos interesa resaltar la importancia de la unidad del equipo directivo (Álvarez, 2010) y la apertura a las distintas propuestas del conjunto de profesores en el marco de la participación.

\section{Procesos de retroalimentación y autoevaluación}

El profesorado parece incidir en una queja respecto a la manera en la que normalmente se desarrollan; ya que estos procesos forman parte de las estructuras normativas emanadas por la Administración y no forman parte de la cultura de los centros para la mejora de los procesos. La evaluación interna o autoevaluación se realiza en los centros educativos respondiendo a una "imposición" marcada en la norma. Ello trae como consecuencia la falta de eficacia de los programas de mejora (Gairín Sallán, 1995; LOMCE, 2013: art. 27) que se diseñan tomando como criterio los resultados de la evaluación interna y, por consiguiente, la ineficacia de los mismos para la mejora continua. Además, el profesorado hace hincapié en la figura del ETCP; lo define como "palanca" o "correa transmisora" para potenciar los procesos de retroalimentación entre el equipo directivo y el claustro de profesores. También se insiste en la necesidad de participación de otros órganos y organizaciones que forman parte de la comunidad educativa.

\section{Resultados e intercambio de una BP}

Hay que decir que todo centro es diferente y por ello singular. Cada profesor, al llegar a un centro, incorpora su cultura (Owens, 1976) y el centro se verá influenciado por la cultura de este; por tanto aquellas BB. PP. que den fruto en un centro respecto a las formas de dirigir y organizar pudieran no dar el mismo resultado en otro. Así pues, el profesorado participante subraya la necesidad de la "exportabilidad" de la BP; para ello es necesario que el propio equipo directivo genere confianza en la innovación de propuestas o formas de realización. Coincidimos en las investigaciones que establecen las características que deben tener las BB. PP. (UNESCO, 2009; Pérez, 2011; Zabalza, 2012; Castillo et al., 2017), en el sentido de que una BP debe ser transferible a otras situaciones y al mismo tiempo pueda ser visibilizada. Destacamos un requisito para que se dé una $\mathrm{BP}$ según el profesorado: "Una BP no solo debe conseguir buenos resultados, sino mejorar los resultados anteriores". De igual forma, Giménez et al. (2003: 5-6) contribuyeron con distintos criterios de definición de una BP, entre ellos, la replicabilidad. 


\section{Respuesta a situaciones novedosas}

El equipo directivo deberá armonizar entre dos polos separados: la mediación y la aplicación del marco normativo, ya que entre sus funciones se encuentra la de "garantizar el cumplimiento de la ley" (LOE, 2006: art. 132). También queremos incidir en la necesidad del conocimiento profundo del contexto en el que se ubica el centro por parte del director; en este sentido, la normativa por la que se selecciona a los directores valora de manera muy positiva este conocimiento (Batanaz, 2006; Silva et al., 2017).

\section{Variables a tener en cuenta para alcanzar objetivos}

Según el estudio, estas variables se centran en la creación de un clima positivo, conocimiento del contexto intraescolar y extraescolar y la construcción de relaciones positivas. Este tipo de variables coinciden con las de investigaciones anteriores (De Pablos y González, 2007), que hacían referencia a "el clima de colaboración y participación que debe producir en el seno de los centros"; así como con Ritacco y Amores (2011) que destacan el "clima y la convivencia" como variables fundamentales para alcanzar un objetivo.

\section{Actitudes del director}

A los directores se les está exigiendo un rol no solo pedagógico, sino de gestión de la información, análisis, razonamiento crítico, comunicación oral y escrita, negociación, conciliación y toma de decisiones, etc. Los profesores participantes remarcan otras cualidades: "Debe ser buen compañero, con capacidades mediadoras, empático, reflexivo, objetivo, paciente, resolutivo, que sea un modelo de conducta para el resto de la comunidad educativa, que muestre una actitud positiva hacia el trabajo y que esté informado, que esté al día sobre lo que sucede en su centro".

\section{Dinámicas de proyecto e innovación}

Nos parece que una de las características más importantes de la definición de BB. PP. es la relación que tiene con la innovación. La figura del director debe ser el de un profesional comprometido con la posibilidad de dinamizar el centro, ejercer el liderazgo pedagógico (Bolivar, 2013; Serrano et al., 2017) e incluir nuevas formas de hacer que generen mejoras y al mismo tiempo se divulguen como apertura al exterior.

En síntesis, llegamos a las siguientes conclusiones respecto a la consideración de las BB. PP. en la organización, gestión y dirección de los centros educativos:

a) Las relaciones con la comunidad educativa deben ser respetuosas, bidireccionales y en la mayoría de las ocasiones horizontales.

b) La autoevaluación debe ser considerada como una tarea normalizada con el objetivo de la mejora continua, no de rendir cuentas.

c) Compartir las BB. PP. con otros centros, y a su vez implementar aquellas que hayan dado buenos resultados, aplicando los coeficientes de corrección apropiados.

d) Ante situaciones novedosas, no previsibles, actuar desde un enfoque mediador con una visión de respeto al marco normativo si existiera en la disciplina en cuestión.

e) Tener presente el contexto intra y extraescolar, generando y construyendo relaciones positivas de colaboración y participación.

f) El director debe tener competencias para el desarrollo de BB. PP., relacionadas con comunicación oral y escrita, negociación, conciliación, mediación, etc.

g) La innovación y la dinamización de prácticas educativas que den respuesta a la mejora continua deben ser los pilares fundamentales en los que se sustente la planificación y organización docente. 


\section{Referencias bibliográficas}

Abdoulaye, A. (2003). Conceptualisation et Dissemination des Bonnes Pratiques en Éducation: Essai d'une Approche Internationale à Partir d'enseignements Tirés d'un Projet. Ginebra: Bureau International d'Education.

Alexander, R. (1997). Policy and practice in primary education: local initiative, national agenda. Londres: Routledge.

Álvarez, M. (2010). Liderazgo compartido: buenas prácticas de dirección escolar. Madrid: Wolters Kluwer Educación.

Aracil, J. (1986). Máquinas, sistemas y modelos. Un ensayo sobre sistemática. Madrid: Tecnos.

Barrios, C., Iranzo, P., Tierno, J. y Camarero, M. (2015). Modelos y funciones de dirección escolar en España: el caso de Tarragona. Revista Iberoamericana de Educación, 67, 89-106.

Batanaz, L. (2006). La función directiva, elemento clave para el desarrollo de las instituciones educativas. Revista de la Asociación de Inspectores de Educación (ADIDE). Avances en supervisión educativa, 4.

Bernal, J. L. (1995). La satisfacción de los directivos con su trabajo: su autoestima. Organización y Gestión Educativa, 5, 3-7.

Bertalanffy, L. (1976). Teoría general de sistemas. México: Fondo de Cultura Económica de España.

Blase, J. y Kirby, P. (2013). Estrategias para una dirección escolar eficaz: cómo motivar, inspirar y liderar. Madrid: Narcea.

Bolívar, A. (2013). La dirección escolar en España: de la gestión al liderazgo. Ponencia presentada en el Congreso Internacional sobre Dirección de Centros Educativos. Universidad de Deusto, Donosti, 1 al 3 julio de 2013.

Cantón, I. (2013). Antecedentes, selección, formación y calidad de los directores escolares. Participación educativa, 3, 165-173.

Carmona Márquez, F., Ramos Corpas, M. J. y Castillo García, M. (2014). Una buena práctica en un IES: la organización de las sesiones de evaluación del alumnado. En Libro de Actas INNOVAGOGIA, 350-363. Editorial AFOE Formación.

Castillo García, M. (2015). El rol de las buenas prácticas educativas en los centros docentes: organización, funcionamiento y docencia. Tesis Doctoral. Departamento de Educación y Psicología Social. Universidad Pablo de Olavide, Sevilla.

Castillo García, M., Ramos Corpas, M. J. (2012). Buenas prácticas en la atención a la diversidad. Una aproximación teórica. Cádiz: VII Congreso de ADIDE Andalucía.

Castillo García, M., Ramírez-Fernández, M. B. y Ramos Corpas, M. J. (2015). El panorama de la investigación en buenas prácticas TIC. Innovaciones con tecnologías emergentes. Universidad de Málaga.

Castillo García, M., Amador Molina, L., Ramos-Corpas, M. J. y Musitu Ochoa, G. (2017). El rol de buenas prácticas educativas (identidad docente). Editorial Académica Española.

Chickering, A. y Gamson, Z. (eds.) (1991). New directions for teaching and learning: aplying the seven principles for good practice in undergraduate education. Nueva York: Jossey-Bass.

Colom, A. J. (1970). Sociología de la educación y teoría general de sistemas. Barcelona: Oikos-Tau.

Creswell, J. W. y Miller, D. L. (2000). Determining validity in qualitative inquiry. Theory into Practice, 39(3), 124-131. doi: 10.1207/s15430421tip3903_2

De Pablos, J. y Jiménez, R. (2007). Buenas prácticas con TIC apoyadas en las políticas educativas: claves conceptuales y derivaciones para la formación en competencias ECTS. Revista Latinoamericana de Tecnología Educativa, 6(2), 15-28. 
Epper, R. y Bates, A. W. (2004). Enseñar al profesorado cómo utilizar la tecnología. Buenas prácticas de instituciones líderes. Barcelona: Editorial UOC.

Escudero, J. M. (2009). Buenas prácticas y programas extraordinarios de atención al alumnado en riesgo de exclusión educativa. Profesorado. Revista de Currículum y Formación del Profesorado, 13(3), 107-141.

Fullan, M. (2014). The principal: three keys to maximizing impact. Nueva York: Jossey-Bass.

Gairín, J. (1995). Estudio de las necesidades de formación de los equipos directivos de los centros educativos. Madrid: MEC-CIDE.

Glaser, B. y Strauss, A. (1967). The discovery of grounded theory: strategies for qualitative research. Nueva York: Aldine Publishing Company.

Informe McKinsey \& Co. (2010). How the world's most improved school systems keep getting better. Recuperado de http://agaeve.ced.juntaandalucia.es/educacion/agaeve/docs/Como_continuan_mejorando_los_sistemas_educativos de_mayor_progreso_en_el_mundo_Informe_McKinsey_2010_ingles-.pdf

Iranzo, P., Tierno, J. y Barrios, R. (2014). Autoevaluación institucional y dirección de centros inclusivos. Teoría de la educación. Revista Universitaria, 26(2), 229-257.

Lorente, A. (2012). Apuntes sobre "el desarrollo profesional" de los directores de los centros docentes. Fórum Aragón, 5, 23-27.

Marqués, P. (2002). Buenas prácticas docentes. Revista DIM.

Mayorga, A. (2007). La dirección educativa y su problemática. CEE Participación Educativa, 5, 93-97.

MECD (2013). Ley Orgánica 8/2013, de 10 de diciembre, de mejora de la calidad de la educación (BOE de 9 de diciembre de 2013).

MECD (2014). Real Decreto 894/2014, de 17 de octubre, por el que se desarrollan las características del curso de formación sobre el desarrollo de la función directiva establecido en el artículo 134.1.c) de la Ley Orgánica 2/2006, de 3 de mayo, de Educación, así como de los correspondientes cursos de actualización de competencias directivas.

Montero Alcaide, A. (2012). Selección y evaluación de directores de centros educativos. Madrid: Wolters Kluwer Educación.

Murillo, F. J. (2008). Hacia un modelo de eficacia escolar. Estudio multinivel sobre los factores de eficacia de las escuelas españolas. REICE: Revista Electrónica Iberoamericana sobre Calidad, Eficacia y Cambio en Educación, 6(1), 4-28.

Murillo, F. J. y Gómez, J. C. (2006). Pasado, presente y futuro de la dirección escolar en España. REICE, 4(4e), 85-98.

Olsen, W. (2004). Triangulation in social research: qualitative and quantitative methods can really be mixed. En M. Holborn (ed.), Development in Sociology. Ormskirk, Inglaterra: Causeway Press.

Owens, R. G. (1976). La escuela como organización: tipos de conducta y práctica organizativa. Madrid: Santillana.

Pérez, G. (2011). Buenas prácticas en las universidades para adultos. Revista de Ciencias de la Educación, 207-226.

Ramos-Corpas, M. J. (2013). Convivencia escolar y buenas prácticas. Málaga: IC Editorial.

Ramos Corpas, M. J. y Castillo García, M. (2013). Buenas prácticas en los institutos de educación secundaria. Una práctica orientada a la utilización de la técnica sociométrica para profundizar en el conocimiento del alumnado. Avances en Supervisión Educativa: Revista de la Asociación de Inspectores de Educación de España, 18.

Ritacco, M. J. y Amores, F. J. (2011). Buenas prácticas en las distintas medidas de prevención del fracaso escolar en la Comunidad Autónoma de Andalucía. Revista Latinoamericana de Inclusión Educativa, 5(1), 161-181. Recuperado de http://www.rinace.net/rlei/numeros/vol5-numl/art10.pdf 
Rodríguez, J., Rodríguez, A., Artiles, J., Aguiar, M. y Alemán, J. (2013). El acceso a la dirección escolar: dificultades y necesidades. Educar, 49(1), 105-125.

Serrano Albendea, R. y Martín-Cuadrado, A. M. (2017). Diagnosis de la problemática actual de las direcciones escolares. Revista Complutense de Educación, 28(4).

Silva, P., del Arco, I. y Flores Alarcia, O. (2018). La formación de directores escolares en Cataluña. Lecciones aprendidas a cinco años del decreto de dirección. Bordón. Revista de Pedagogía, 70(1), 109-124. doi: https://doi.org/10.13042/Bordon.2017.49427

Strauss, A. y Corbin, J. (1998). Fundamentos de investigación cualitativa. Técnicas y procedimientos para desarrollar teoría enraizada. Nueva Delhi: Sage Publications.

Suárez, C., Del Moral, G. y González, M. T. (2013). Consejos prácticos para escribir un artículo cualitativo publicable en psicología. Psychosocial Intervention, 22(1),71-79.

Thomas, S. P. (2011). Conceptual debates and empirical evidence about the peer review process for scholarly journals. Journal of Professional Nursing, 27(3), 168-173.

UNESCO (2009). Buenas prácticas para una educación de calidad. París: UNESCO.

Valles, M. S. (2003). Técnicas cualitativas de investigación social. Reflexión, metodología y práctica profesional. Madrid: Síntesis.

Vera, J. M., Mora, V. y Lapeña, A. (2006.) Dirección y gestión de centros docentes. Barcelona: Graó.

Villa Sánchez, A., Villardón Gallego, L., Villar Angulo, L. M., de Vicente Rodríguez, P., Borrell, N., Laffitte Figueras, R. y Gairín Sallán, J. (1998). Principales dificultades de la dirección de centros educativos en los primeros años de ejercicio. España: ICE-Universidad de Deusto.

Zabalza, M. A. (2012). El estudio de las "buenas prácticas" docentes en la enseñanza universitaria. Revista de Docencia Universitaria. REDU, 10(1), 17-42. Recuperado de http://redaberta.usc.es/redu

\section{Abstract}

Best practice in the organization, management, and direction of educational centers

INTRODUCTION. The objective of this paper is to deepen the knowledge of best practices in the exercise of school based management by considering the following variables: the relationship between teachers and the leadership team, the feedback processes, the results of a good practice, the response to novel situations, the variables to be taken into account to achieve the objectives, the attitudes of the good Head, the dynamics of project and innovation, the exchange of teaching practices and finally the self-evaluation processes. Numerous studies have been carried out on school leadership in recent decades, although this research provides a new approach such as that of best practices in school leadership. METHOD. A qualitative methodology based on Grounded Theory is used from the information obtained by means of Focus Group and subsequent coding and triangulation of data, with the support of Atlas ti 5.0. RESULTS. The results are presented with specific illustrations according to each of the variables studied. DISCUSSION. It is also organised according to the variables studied and it becomes clear, as the most outstanding aspects, that the relations between the teachers and the leadership team must be respectful, horizontal, bidirectional and participatory, developed through cooperation and collaboration strategies. They must take into account feedback processes by enhancing the culture of self-evaluation as a driver for the improvement processes at the school; and they should not only focus on achieving good results, but also on improving the previous ones; furthermore, the context for its application will be necessary to bear in mind.

Keywords: School based management, Best practices, Feedback, Innovation. 


\section{Résumé}

Bonnes pratiques pour l'organisation, gestion et direction des établissement scolaires

INTRODUCTION. Ce travail a pour objectif approfondir la connaissance des bonnes pratiques dans la direction des centres scolaires et nous avons tenu en compte les suivantes variables: le lien entre les enseignants et l'équipe de direction, le feedback, les résultats positifs suivant une bonne pratique, la réussite dans des situations nouvelles, les changements à tenir en compte pour atteindre les objectifs, les qualités d'un bon directeur, la gestion de projets et l'innovation, le pôle de partage des pratiques pédagogiques et le processus d'auto-évaluation. Même s'il y a des nombreuses études par rapport à la direction des établissements, cette investigation apporte une nouvelle perspective : les bonnes pratiques dans la direction des établissements éducatifs. MÉTHODE. La méthodologie est qualitative, elle est fondée sur la Grounded Theory avec l'information obtenu grâce au Grupo Focal et le cryptage postérieur et la triangulation des données à l'aide de Atlas ti 5.0. RÉSULTATS. Notre étude s'appuie sur des illustrations spécifiques concernant chaque variable, déjà étudiés pour présenter des résultats. DISCUSSION. Il est organisé en fonction des variables déjà étudiés et souligne, comme principaux points : l'importance des relations entre les enseignants et l'équipe de direction, devant être respectueuses, horizontales, bidirectionnelles et participatives, développées grâce à stratégies de coopération et collaboration. Il faut tenir en compte l'importance du feedback comme véhicule des processus de modernisation dans les centres éducatifs en tant que produit du renforcement de la culture de l'auto-évaluation. Il est important pas seulement d'obtenir des bons résultats mais aussi de les améliorer dans des occasions ultérieures. Pour cela il faudra prendre en considération le contexte mis en ouvre.

Mots-clés: Direction d'établissement scolaires, Bonne pratiques, Feedback, Innovation.

\section{Perfil profesional de los autores}

\section{Manuel Jesús Ramos Corpas (autor de contacto)}

Doctor y profesor en la Universidad Pablo de Olavide de Sevilla, en el Área de Psicología Social. Ha sido director de IES en varios centros y actualmente es inspector de educación. Es integrante del grupo interuniversitario de investigación Lisis, especializado en el estudio de la violencia durante la etapa adolescente. Ha coordinado diversos proyectos de investigación. Ha participado en el proyecto de investigación Buenas Prácticas en la Organización y Funcionamiento de los Institutos de Educación Secundaria, aprobado por la Consejería de Educación de la Junta de Andalucía.

Correo electrónico de contacto: mjramcor@upo.es

Dirección para la correspondencia: Edificio 14, 2. ${ }^{a}$ planta, despacho n. ${ }^{\circ} 11$. Ctra. Utrera, km 1, 41013 Sevilla (España).

\section{Manuel Castillo García}

Doctor por la Universidad Pablo de Olavide (Sevilla). Ha sido profesor de enseñanza secundaria y director de IES, con formación de arquitecto, ingeniero y pedagogo. Es inspector de educación y profesor asociado de la Universidad de Sevilla. En los últimos años ha desarrollado una línea de 
investigación sobre buenas prácticas en los centros educativos, lo que le ha permitido presentar varias comunicaciones a diferentes congresos nacionales e internacionales y publicar artículos en diversas revistas científicas del ámbito de la educación.

Correo electrónico de contacto: m.castillo23@us.es

\section{Gonzalo del Moral Arroyo}

Doctor en Psicología por la Universidad Pablo de Olavide y terapeuta familiar. Es integrante del grupo interuniversitario de investigación Lisis, especializado en el estudio de la violencia durante la etapa adolescente. Ha publicado diversos artículos científicos, libros y capítulos de libros centrados en dos temática principales: la violencia escolar entre iguales y la violencia filio-parental. Del mismo modo, desde 2008 trabaja en colaboración con los centros de formación del profesorado de Andalucía Occidental formando a profesorado y padres y madres en resolución de conflictos, mediación e inteligencia emocional, así como con servicios sociales y diputaciones andaluzas para la formación del personal técnico en el abordaje terapéutico de la violencia filio-parental.

Correo electrónico de contacto: gmorar@upo.es 